\title{
CARACTERÍSTICAS DE LA DEMANDA NO URGENTE EN EL SERVICIO DE EMERGENCIA DE UN HOSPITAL DE LA SEGURIDAD SOCIAL EN EL PERÚ
}

\author{
CHARACTERISTICS OF THE NON-URGENT DEMAND IN THE EMERGENCY SERVICE OF A SOCIAL \\ SECURITY HOSPITAL IN PERU \\ Waldo Taype-Huamaní ra, Lucila De la Cruz-Rojas²,b, Delcio Miranda-Soler ${ }^{1, a}$, José Amado-Tineo ${ }^{1, a, c, d}$
}

\begin{abstract}
RESUMEN
Objetivo: Describir las características de pacientes con demanda no urgente en el departamento de emergencia de un hospital urbano de la seguridad social en el Perú. Métodos: Estudio observacional realizado en un hospital nacional de referencia. Muestreo aleatorio estratificado en una semana, 120 pacientes mayores de 14 años de prioridad IV (signos vitales estables, sin riesgo de complicación inmediata y de consulta externa general). Se aplicó un cuestionario prediseñado validado. Resultados: El $66 \%$ de pacientes fue de sexo femenino. Las edades más frecuentes fueron mayores de 65 años y el grupo de 20 a 40 años. El $47 \%$ fueron trabajadores activos, con nivel de instrucción secundaria o superior en $86 \%$ de casos. El $83 \%$ contaban con teléfono celular personal y el $73 \%$ con televisión por cable o internet en su domicilio. El 63\% llegó a emergencia en transporte público. El tiempo de demora hasta el hospital fue 3060 minutos en el $50 \%$ de casos y < 30 minutos en $28 \%$. El $33 \%$ no conocía su centro de atención primaria y el $87 \%$ no acudió a otro establecimiento de salud previo a su arribo a emergencia. Los síntomas más frecuentes incluyeron malestar general, odinofagia, diarrea y lumbalgia. El tiempo de enfermedad fue de horas a días en el $91 \%$ de casos. Conclusión: Los pacientes de consulta no urgente que acuden al servicio de emergencia son predominantemente de sexo femenino, adultos jóvenes y mayores, laboralmente activos, con alto nivel educativo y acceso a tecnología de comunicaciones, residentes en áreas cercanas al hospital y sin conocimiento de su centro de atención primaria.
\end{abstract}

Palabras clave: Necesidades y demandas de servicios de salud; Servicios médicos de urgencias; Triaje. (fuente: DeCS BIREME)

\begin{abstract}
Objective: To describe the characteristics of patients with non-urgent demand to the emergency room of an urban social security hospital. Methods: Observational study in a referral hospital. Stratified random sampling, 120 patients older than 14 years of priority IV (patients without commitment to vital functions or risk of immediate complication, which can be attended in outpatient room or decentralized offices). A validated predesigned questionnaire was applied. Performing descriptive statistics with IBM SPSS 24. Results: $66 \%$ of patients were female, most frequent ages over 65 years and between $20-40$ years. $47 \%$ were active workers, with high school level or higher $86 \%$, they had $83 \%$ personal cell phone and $73 \%$ wire TV or internet at home. $63 \%$ arrived at the public transport emergency, the delay time to the hospital was 30-60 minutes in $50 \%$ of cases and $<30$ minutes in $28 \%$. He did not know his primary care center $33 \%$, he was never treated before in this $57 \%$ and did not go to another health facility prior to his $87 \%$ emergency arrival. Most frequent symptoms: malaise, odynophagia, diarrhea and low back pain; with presentation time between hours and days in $91 \%$ of cases. Conclusion: Non-urgent consultation patients in the emergency room are predominantly female, young and old people, who are active in work, with a high level of education and access to communications technology, reside near the hospital and do not know their primary care center.
\end{abstract}

Key words: Health services needs and demand; Emergency medical services; Triage room. (source: MeSH NLM)

${ }^{1}$ Hospital Nacional Edgardo Rebagliati Martins-EsSalud, Lima-Perú.

2 Universidad Nacional Federico Villarreal. Lima-Perú.

${ }^{3}$ Universidad Nacional Mayor de San Marcos. Lima-Perú.

a Departamento de Emergencia. Lima-Perú.

b Unidad de Posgrado.

c Facultad de Medicina.

d Médico Internista.

Citar como: Waldo Taype-Huamaní, Lucila De la Cruz-Rojas, Delcio Miranda-Soler, José Amado-Tineo. Características de la demanda no urgente en el servicio de emergencia de un hospital de la seguridad social en el Perú. Rev. Fac. Med. Hum. Octubre 20120; 20(1):20-26. DOI 10.25176/RFMH.v20i1.2544

Artículo publicado por la Revista de la Facultad de Medicina Humana de la Universidad Ricardo Palma. Es un artículo de acceso abierto, distribuído bajo los términos de la Licencia Creative Commons: Creative Commons Attribution 4.0 International, CC BY 4.0 (https://creativecommons.org/licenses/by/4.0/), que permite el uso no comercial, distribución y reproducción en cualquier medio, siempre que la obra original sea debidamente citada. Para uso comercial, por favor póngase en contacto con revista.medicina@urp.pe 


\section{INTRODUCCIÓN}

La demanda de atención en el servicio de emergencia se ha incrementado en las últimas décadas en todo el mundo. ${ }^{(1-3)} \mathrm{El}$ incremento de la demanda de atención en emergencia en hospitales de referencia se explica por incremento de la población, mayor prevalencia de enfermedades crónicas, deficiencias del sistema sobretodo en la atención primaria y a la preferencia de la población de establecimientos de salud de mayor complejidad y tecnología de punta. El hacinamiento de estos servicios se ha constituido en un problema prioritario de investigación. . $^{(4)}$

Una medida ante esta sobredemanda y riesgo en el servicio fue implementar los sistemas de triaje estructurados. Desde el 2008 el sistema de seguridad social peruano (EsSalud) adopta el triaje de emergencia de 4 prioridades, donde la prioridad I requiere resucitación inmediata, prioridad II corresponde a una emergencia y prioridad III a una urgencia. La prioridad IV corresponde a pacientes sin compromiso de funciones vitales ni riesgo de complicación inmediata, que puede ser atendido en consulta externa o consultorios descentralizados (es decir no constituye urgencia ni emergencia). ${ }^{(6)}$

Existen porcentajes variables de consulta inadecuada en los servicios de emergencia, entre 8 y $62 \%$ reportados en distintas partes del mundo, con una mediana de $37 \% .{ }^{(7-10)}$ En nuestro país se presenta una situación similar en todos los subsistemas de salud: ministerio de salud, fuerzas armadas y policiales e incluso en el sector privado. ${ }^{(11,12)}$ En la seguridad social el problema es mayor en los hospitales de referencia de cada red asistencia. ${ }^{(13,14)}$

La consulta inadecuada en emergencia está influenciada por diversos factores y exponen a una distribución inadecuada de los recursos que casi siempre son escaso y más aún a la atención inadecuada de las verdaderas emergencias. ${ }^{(15-18)}$ Es por eso que la presente investigación buscó describir las características de los pacientes con demanda no urgente al servicio de emergencia de un hospital urbano de la seguridad social.

\section{MÉTODOS}

Se realizó un estudio observacional en el servicio de emergencia de adultos del hospital nacional Edgardo Rebagliati Martins de EsSalud, ubicado en Jesús María, Lima-Perú en el mes junio del año 2014, no incluyendo emergencias pediátricas, ginecológicas $\mathrm{ni}$ psiquiátricas.

Se consideró demanda no urgente a emergencia a las consultas consideradas de prioridad IV (pacientes con signos vitales estables, sin riesgo de complicación inmediata y que pueden ser atendidos por consulta externa general) según evaluación inicial en el tópico de triaje, en pacientes mayores de 14 años que acudieron a dicho servicio.

Se incluyó 120 pacientes, aplicando muestreo aleatorio estratificado de lunes a viernes de una semana, calculado con fórmula de muestreo para determinar proporciones, considerando $p=0,50$, intervalo de confianza $95 \%$, tolerancia de error $5 \%$ y tamaño poblacional de 608 pacientes de prioridad IV para una semana laborable.

Después de ser evaluados en tópico de atención rápida de del mismo servicio, se aplicó un cuestionario adaptado de un instrumento para utilización inadecuada del servicio de urgencias que incluyó factores predisponentes, facilitadores y de necesidad; así como la percepción del servicio recibido, ${ }^{(19,20)}$ validado por opinión de dos expertos locales (Tabla 1).

Los datos fueron codificados y procesados en Microsoft Excel 2010. Realizándose análisis estadístico descriptivo utilizando el programa estadístico IBM SPSS 24.0. Se siguió principios de buenas prácticas de investigación con la autorización de la jefatura del servicio.

\section{RESULTADOS}

Las características de los pacientes se presentan en la Tabla 2. El grupo etáreo más frecuente, según la clasificación de la Organización Mundial de la Salud, fue mayor de 65 años 34\% y entre 20-40 años 29\% (Figura 1). La situación laboral más frecuente fue trabajador activo. En cuanto a grado de instrucción, tuvo educación secundaria o superior el $86 \%$ de pacientes.

En lo referente a acceso a transporte, telecomunicaciones y tecnología, contaba con vehículo propio $7 \%$, teléfono celular personal $83 \%$ y televisión por cable o internet en su domicilio $73 \%$. Llegó a la emergencia en transporte público $63 \%$ y en taxi $25 \%$. El tiempo de demora para llegar hasta el hospital fue 30-60 minutos en el 50\% de casos y $<30$ minutos en $28 \%$ (Tabla 2).

El 33\% de pacientes mencionó que no conocía su centro de atención primaria, el $57 \%$ no se atendió nunca antes en este y $87 \%$ no acudió a otro establecimiento de salud previo a su arribo a emergencia. El $72 \%$ de pacientes vino a la emergencia desde su domicilio y el $17 \%$ desde su centro de trabajo (Tabla 2).

Los síntomas más frecuentes fueron: malestar general, odinofagia, diarrea y lumbalgia. La duración de estos síntomas fue entre horas y días en el $91 \%$ de los casos. 
Acudió a emergencia por la rapidez de atención 33\% y por problemas administrativos del sistema de salud $36 \%$, en otros menciona que acudió por comodidad el $8 \%$ de casos. Casi todos los pacientes incluidos en el estudio, recomendaría el servicio a sus familiares o conocidos (Tabla 2).

Tabla 1. Estructura del cuestionario para evaluar demanda no urgente al servicio de emergencia de un hospital urbano de la seguridad social 2014.

Factores

Preguntas del cuestionario

Factores predisponentes

Edad

1. Marque la respuesta entre que edades se encuentra usted

Sexo

2. Marque el sexo que le caracteriza

Característica familiar

3. Marque la característica familiar que le representa

Situación laboral

4. Marque la situación laboral que le caracteriza

5. Marque la ocupación laboral que le caracteriza

Nivel de estudio

6. Marque su nivel de estudio

Factores facilitadores

7. Dispone de vehículo propio

Nivel económico

8. Dispone de teléfono celular

9. En su domicilio cuenta con internet y cable

10. Como se traslada usted para acudir emergencia del hospital

11. El centro de atención primaria donde usted se atiende corresponde a la red de salud

Lugar de adscripción

12. ¿Cuál es su centro de atención primaria de procedencia?

13. ¿Antes de acudir a emergencia del hospital se atendió en su centro de atención primaria?

14. ¿Antes de acudir a emergencia del hospital fue a otro centro de salud?

Accesibilidad

15. ¿De dónde viene a emergencia del hospital para ser atendido?

16. ¿Cuánto tiempo demora usted para llegar a emergencia del hospital?

Factores de necesidad

17. ¿Qué síntoma presentó cuando acudió a emergencia del hospital?

Percepción del estado de salud

18. ¿Cómo se siente usted?

19. Tiempo de inicio del síntoma

Expectativas de los usuarios

20. Motivo por el cual acude a emergencia del hospital

21. ¿Recomendaría este servicio a sus familiares o amistades?

Fuente. Adaptado de Carpio Pérez A. Estimación de la utilización inadecuada del servicio de urgencias de un hospital universitario. Salamanca; Tesis doctoral 2008 y Sánchez-López J. Factores asociados al uso inadecuado de un servicio de urgencias hospitalario. Emergencias 2005;17:138-44 
Tabla 2. Características de los pacientes con demanda no urgente al servicio de emergencia de un hospital urbano de la seguridad social 2014.

\section{Características}

\section{$N(120)$}

Sexo femenino

79

66

Edad $>65$ años

41

34

Característica familiar:

- vive solo

- en familia

- en casa de reposo

5

4

Situación laboral:

- activo

- jubilado

- ama de casa

24

20

Nivel de estudios:

- Primaria

- Secundaria

- Superior técnica

- Superior universitaria

Tiene vehículo propio

Tiene teléfono celular o cable

En que se trasladó a emergencia:

- transporte público

- taxi

- otros

Conoce su centro de atención primaria

Su centro pertenece a la Red

Acudió a otro centro antes de venir

De qué lugar vino a la emergencia:

- domicilio

52

43

- centro de trabajo

- acompañando a un paciente

- otro

Tiempo en llegar a emergencia:

$-<30$ minutos

$-30-60$ minutos

- > 60 minutos

Se siente con buen estado de salud

Tiempo de inicio de síntomas:

- horas

- días

- semanas

Motivo por el que acude a emergencia:

- rapidez en la atención

- espera hospitalización

- demora en cita para consultorio 


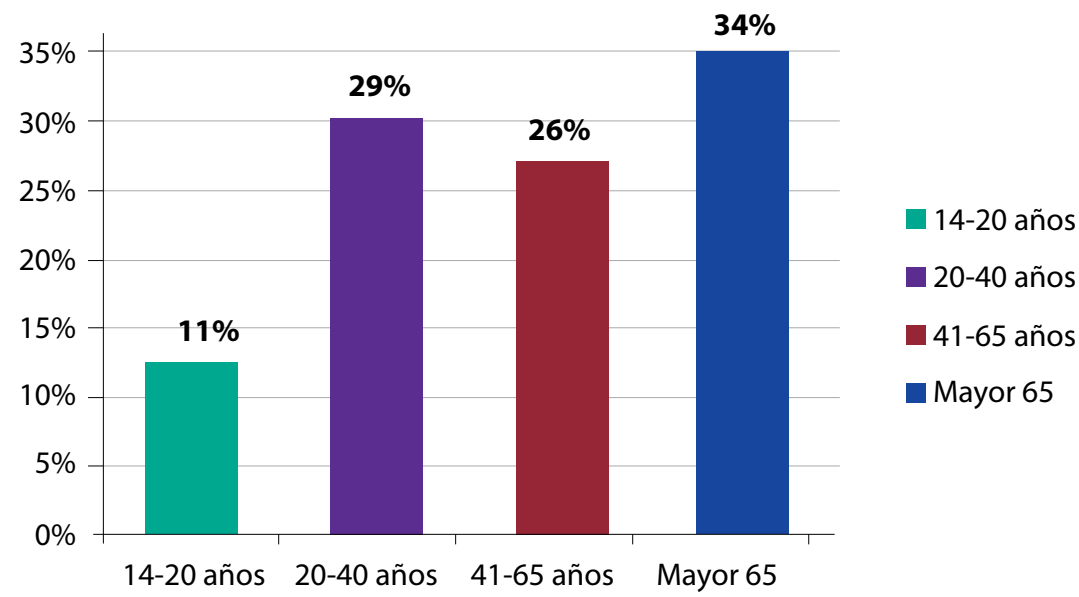

Figura 1. Grupo etario de pacientes con consulta inadecuada al servicio de emergencia de un hospital urbano de la seguridad social 2014.

\section{DISCUSIÓN}

El estudio evaluó múltiples factores relacionados al uso inadecuado de los servicios de emergencia; entre los que se encuentran sexo, edad, condición laboral, grado de instrucción, acceso a tecnología, accesibilidad espacio-temporal, conocimiento del sistema de salud e implementación de este en la comunidad ${ }^{(2,3,16)}$.

El crecimiento de la demanda de atención en los servicios de emergencia ha sido progresivo, pero cuando diferenciamos por prioridades observamos que el grupo que más creció fue el de Prioridad IV y en forma logarítmica es decir los que hacen uso inadecuado del servicio de emergencia ${ }^{(13,14)}$.

En la actualidad, los servicios de Urgencias Emergencias Hospitalarias se enfrentan a nuevos escenarios asistenciales que influye en la utilización repetida de los servicios de salud; esto es debido, por un lado, al envejecimiento de la población que conlleva el agravamiento como consecuencia de la edad de distintas patologías físicas así como diferentes problemas sociales (los ingresos económicos reducidos, el bajo nivel educacional, la soledad, etc.) y por otro, al aumento de los movimientos migratorios, sin olvidar también, otros factores asociados a la conducta de los usuarios ${ }^{(9,16)}$.

Entre los factores predisponentes en el uso inadecuado del servicio de emergencia se reporta que en la edad predomina la población más joven, en la situación familiar predominan los pacientes que viven con su parentela y la demanda directamente proporcional al mejor nivel de instrucción. Entre los factores facilitadores en el uso inadecuado del servicio de emergencia, con respecto a la distancia predomina en los pacientes que viven más cerca, usuarios de Cable e internet y más del $50 \%$ ya han sido evaluados en sus policlínicos. La ausencia de barreras administrativas en el servicio de emergencia y carencia de oferta de citas en consultorios externos son dos caras de la misma moneda que facilitan la demanda de atención inadecuada en el servicio de emergencia ${ }^{9,16,19,20}$.

Al analizar factores específicos, podemos decir que el sexo femenino fue más frecuente entre los pacientes con uso inadecuado de los servicios de emergencias, lo que coincide con múltiples estudios publicados, con valores de riesgo relativo entre 1,12 a 1,56 pero en este factor pueden existir muchas diferencias culturales en distintas partes del mundo $0^{(9,16,19-21)}$.

En lo referente a la edad, varios estudios demostraron que los adultos más jóvenes tenían más probabilidades de tener visitas no urgentes en comparación con los adultos mayores. Sin embargo otros artículos no encontraron asociación entre el uso no urgente de los servicios de emergencias y la edad. Sin embargo en nuestro estudio, a pesar que el grupo adulto mayor es más frecuente, el grupo de adultos jóvenes es proporcionalmente mayor a reportes del total de pacientes que solicitan consulta en emergencia ${ }^{(13,21)}$.

Varios estudios encontraron una asociación directa entre el estado socioeconómico (escolaridad e ingresos) y el uso inapropiado de los servicios de emergencias ${ }^{(9,22)}$. Estudios descriptivos que evaluaron las razones relacionadas con el acceso para preferir los servicios de emergencias detectaron dificultades para consultar la atención primaria: como el cierre de la instalación de atención primaria, la dificultad para obtener una cita y el tiempo de espera como razones para el uso inapropiado ${ }^{(16,22)}$.

Estudios analíticos encontraron una asociación entre el difícil acceso a la atención primaria de salud y el uso inapropiado de los servicios de emergencia. En un estudio de cohorte en los Estados Unidos, esta asociación tuvo un valor de p 0,029. Las variables 
que comprenden este indicador, la dificultad para programar la atención primaria, el contacto telefónico difícil para la atención primaria y el mayor tiempo de espera para una cita de atención primaria también se asociaron con el uso inadecuado de los servicios de emergencias ( $p<0,03$ ) cuando se evaluaron individualmente en el análisis multivariado ${ }^{(23)}$. En un estudio brasileño, la dificultad para obtener una cita de atención primaria, la negativa del médico de atención primaria a tratar a los pacientes sin una cita programada previamente y la atención primaria abierta en horario más corto se asociaron con el uso inapropiado de los servicios de emergencias (riesgo relativo de $1.38,1.44$ y 1.63 , respectivamente) $)^{(16)}$.

También se reporta asociación entre el acceso deficiente (por ejemplo dificultad para obtener atención médica o no tener un médico de cabecera) y el uso no urgente del servicio de urgencias, siendo la barrera más importante para obtener atención fuera del servicio de urgencias era la imposibilidad de obtener una cita en la consulta externa ${ }^{(9,16)}$.

Entre los factores de necesidad en el uso inadecuado del servicio de emergencia, el síntoma principal fue malestar general y el tiempo de evolución fue días, a diferencia de otros estudios principalmente europeos (España) que reportan dolor de horas de evolución ${ }^{(6,15)}$. El $93 \%$ de pacientes de prioridad IV atendidos en emergencia recomendarían la atención del servicio a familiares o amigos; esto representa una dependencia hospitalaria de la población a pesar de no justificarse su atención en ese nivel de complejidad.

Este grupo de pacientes realmente no impacta directamente en el hacinamiento de las salas de observación del servicio de emergencia, pero sí en las salas de espera y requieren la disposición de un lugar adecuado de atención fuera de la estructura de emergencia, idealmente que sean dirigidos a la atención médica ambulatoria de otros niveles de atención.
Entre las limitaciones del presente estudio tenemos una muestra pequeña y sólo se evaluó el grupo de consulta no urgente, no comparándolo con otros. En el momento de recolección de datos estaba vigente el sistema de 4 prioridades, mientras que actualmente la institución utiliza el sistema tipo Manchester de 5 prioridades. Se evaluó a los pacientes después de ser atendidos, probablemente no se haya encuestado a otros que fueron derivados a otros establecimientos de atención primaria sin atenderlos en el servicio. Pero estos datos son importantes porque nos muestran las características de uno de las causas del hacinamiento existente y nos sirven para seguir investigando el tema, en busca de soluciones efectivas.

\section{CONCLUSIÓN}

Finalmente, concluimos que las características de los pacientes que realizan consulta no urgente al servicio de emergencia son: sexo femenino, adultos jóvenes y mayores, laboralmente activos, con alto nivel educativo y acceso a tecnología de comunicaciones, vivir cerca al hospital y con síntomas recientes.

Contribuciones de autoría: Los autores participaron en la génesis de la idea, diseño de proyecto, recolección e interpretación de datos, análisis de resultados y preparación del manuscrito del presente trabajo de investigación.

\section{Financiamiento: Autofinanciado.}

Conflicto de interés: Los autores declaran no tener conflicto de interés en la publicación de este artículo.

Recibido: 07 de junio 2019

Aprobado: 25 de noviembre 2019

Correspondencia: Jose Amado Tineo

Dirección: Jr. Belisario Flores 328 Dpto 301. Lince, Lima, Peru-Lima14

Teléfono: +1990452547

Correo:jpamadot@gmail.com;jamadot@unmsm.edu.pe 


\section{REFERENCIAS BIBLIOGRÁFICAS}

1. Hsu NC, Shu CC, Lin YF, Yang MC, Su S, Ko WJ. Why do general medical patients have a lengthy wait in the emergency department before admission? J Formos Med Assoc 2014;113(8):557-61. DOI: 10.1016 / j.jfma.2012.08.005.

2. Hsu CM, Liang LL, Chang YT, Juang WC. Emergency department overcrowding: Quality improvement in a Taiwan Medical Center. J Formos Med Assoc 2019;118(1P1):186-93. doi: 10.1016/j.jfma.2018.03.008

3. Giunta DH, Pedretti AS, Elizondo CM, Grande Ratti MF, González F, Waisman GD, et al. Descripción de las características del fenómeno Crowding en la Central de Emergencia de Adultos, en un hospital universitario de alta complejidad: estudio de cohorte retrospectiva. Rev Med Chil 2017;145(5):557-63. Disponible en: http://dx.doi.org/10.4067/ S0034-98872017000500001

4. Thompson C, Hayhurst C, Boyle A. How have changes to out-of-hours primary care services since 2004 affected emergency department attendances at a UK District General Hospital? A longitudinal study. Emerg Med J 2010;27(1):22-5. doi: 10.1136/emj.2008.068817

5. Smith J, Keating L, Flowerdew L, O'Brien R, Mclntyre S, Morley R, et al. An Emergency Medicine Research Priority Setting Partnership to establish the top 10 research priorities in emergency medicine. Emerg Med J 2017;34(7):454-6. DOI: 10.1136/ emermed-2017-206702

6. Soler W, Gómez-Muñoz M, Bragulat E, Álvarez A. El triaje: herramienta fundamental en urgencias y emergencias. An Sist Sanit Nava 2010;33(1):55-68. Disponible en: http://scielo.isciii.es/scielo.php?script=sci abstract\&pid=S1137-66272010000200008\&lng=es\&nrm=iso\&tlng=es.

7. Lega F, Mengoni A. Why non-urgent patients choose emergency over primary care services? Empirical evidence and manageria implications. Health Policy (New York) 2008;88(2-3):326-38. DOI: 10.1016 j.healthpol.2008.04.005

8. Hoot NR, Aronsky D. Systematic Review of Emergency Department Crowding: Causes, Effects, and Solutions. Ann Emerg Med 2008;52(2):126136.e1. DOI: 10.1016 / j.annemergmed.2008.03.014

9. Durand A-C, Gentile S, Devictor B, Palazzolo S, Vignally P, Gerbeaux P, et al. ED patients: how nonurgent are they? Systematic review of the emergency medicine literature. Am J Emerg Med 2011;29(3):333-45. DOI: 10.1016 / j.ajem.2010.01.003.

10. Uscher-Pines L, Pines J, Kellermann A, Gillen E, Mehrotra A. Deciding to Visit the Emergency Department for Non-Urgent Conditions: A Systematic Review of the Literature. Am J Manag Care. enero de 2013;19(1):47-59. Disponible en: https://www.ncbi.nlm.nih.gov/pmc/articles/PMC4156292/.

11. Ponce Varillas TI. Hacinamiento en los servicios de emergencia. An Fac med 2017;78(2):218-223. DOI: 10.15381/anales.v78i2.13221

12. Andrade JG. Incidencia de demanda de atención en el servicio de emergencia del HCPNP en situaciones no urgentes. Lima 2002. Tesis
UNMSM. 2012. Disponible en: http://cybertesis.unmsm.edu.pe/handle/ cybertesis/1807

13. Vásquez-Alva $\mathrm{R}$, Amado-Tineo J, Ramírez-Calderón F, VelásquezVelásquez R, Huari-Pastrana R. Sobredemanda de atención médica en el servicio de emergencia de adultos de un hospital terciario, Lima, Perú. An la Fac Med 2016;77(4):379. Dispinible en: http://www.scielo.org.pe/scielo. php?script=sci_abstract\&pid=S1025-55832016000400010\&lng=es\&nrm=i so\&tlng=en

14. Taype-Huamani W, Chucas-Ascencio L, Amado-Tineo J. Hacinamiento del servicio de emergencia de adultos del hospital Rebagliati: Reporte de caso. Rev med Rebagliati 2018;1(2):50-5. Disponible en: https://www. researchgate.net/publication/329963374.

15. Castrillón C, Jaime J, David J, Zapata L, Alicia F, et al. Demandas inadecuadas en urgencias e identificación del uso inapropiado de la hospitalización en el centro piloto de ASSBASALUD ese en Manizales. Arch Med 2009;9(1):25-34.

16. Carret ML, Fassa AG, Kawachi I. Demand for emergency health service: factors associated with inappropriate use. BMC Health Serv Res 2007;7(1):131. doi: 10.1186/1472-6963-7-131

17. Pelegrini AHW, Dos Santos JLG, Marques GQ, Ciconet RM, Lima MAD. Organization of health services attention to emergencies: narrative review. Online Brazilian J Nurs 2010;9(1):88-93. Disponible en: https://doi. org/10.5935/1676-4285.20102888.

18. Lowthian JA, Curtis AJ, Cameron PA, Stoelwinder JU, Cooke MW, McNeil JJ. Systematic review of trends in emergency department attendances: an Australian perspective. Emerg Med J 2011;28(5):373-7. doi: 10.1136/ emj.2010.099226

19. Carpio-Pérez A. Estimación de la utilización inadecuada del servicio de urgencias de un hospital universitario. Tesis doctoral Salamanca; 2008. Dlsponible en: https://dialnet.unirioja.es/servlet/tesis?codigo=75803.

20. Sánchez-López J. Factores asociados al uso inadecuado de un servicio de urgencias hospitalario. Emergencias 2005;17:138-44. Dispoble en: https:// dialnet.unirioja.es/servlet/articulo?codigo $=1230143$.

21. Davis JW, Burns JA, Fujimoto RY, Chan H, Juarez DT. Identifying characteristics of patients with low urgency emergency department visits in a managed care setting. Manag Care 2010;19(10):38-44. Disponible en: https://www.ncbi.nlm.nih.gov/pubmed/21049788.

22. Afilalo J. Nonurgent Emergency Department Patient Characteristics and Barriers to Primary Care. Acad Emerg Med 2004;11(12):1302-10. doi: 10.1197/j.aem.2004.08.032

23. Sarver JH, Cydulka RK, Baker DW. Usual Source of Care and Nonurgent Emergency Department Use. Acad Emerg Med 2002;9(9):916-23. doi: 10.1111/j.1553-2712.2002.tb02193.x

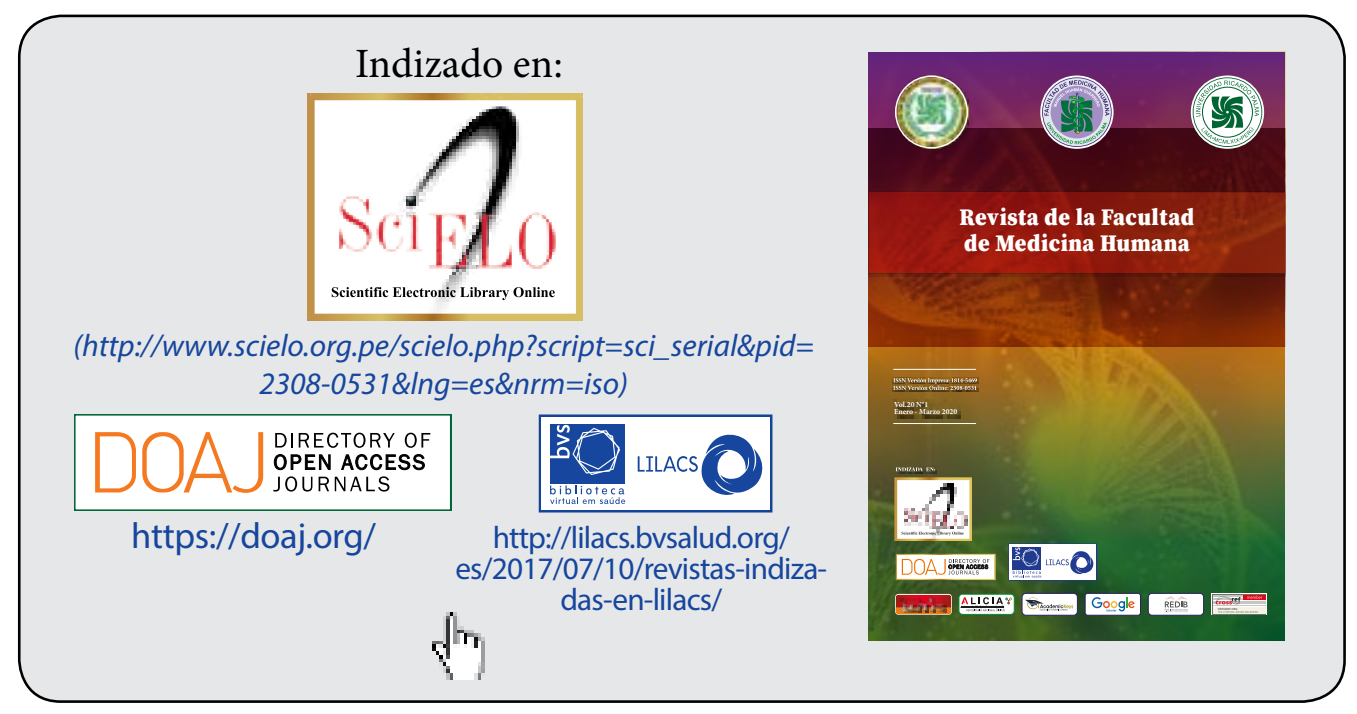

